\title{
ANDES

www.scielo.cl

\section{Ingesta alimentaria de fuentes proteicas en lactantes entre 7-24 meses: tiempo de inicio y cumplimiento de recomendaciones}

\author{
Dietary intake of protein sources in infants between 7-24 months old: start time and \\ compliance with recommendations
}

\author{
Natalia Sepúlveda Alarcóna , Catalina Le Roy Olivos ${ }^{\mathrm{b}}$
}

aResidente, Programa de Especialización en Pediatría, Facultad de Medicina Pontificia Universidad Católica de Chile. Santiago, Chile bDepartamento de Gastroenterología y Nutrición Pediátrica, Facultad de Medicina Pontificia Universidad Católica de Chile. Santiago, Chile

Recibido: 21 de abril de 2020; Aceptado: 1 de octubre de 2020

\section{¿Qué se sabe del tema que trata este estudio?}

Se sabe que existe bajo cumplimiento de recomendación de consumo de alimentos de las guías nacionales, sobre todo en pescado y leguminosas, informado por la Encuesta Nacional de Consumo de Alimentos 2010, sin embargo, se incluyó sólo mayores de 24 meses.

\section{¿Qué aporta este estudio a lo ya conocido?}

Este estudio aporta información de cumplimiento de recomendaciones alimentarias en lactantes entre 7-24 meses, encontrándose que la incorporación de legumbres, huevo y pescado es tardía, el consumo de otras carnes es excesivo, asemejándose al resto de la población chilena.

\section{Resumen}

Una adecuada nutrición en el período de lactante comprende lactancia materna (LM), fórmulas lácteas (FL) e incorporación de alimentación complementaria (AC). Objetivo: Describir el cumplimiento de recomendaciones de ingesta de fuentes proteicas de AC en lactantes sanos entre 7-24 meses. Pacientes y Método: Se estudiaron lactantes sanos de un centro ambulatorio. Para la obtención de las variables en estudio se diseño y aplicó un cuestionario, del cual se obtuvieron: datos demográficos, duración de LM, uso de FL, incorporación y cumplimiento de ingesta para las fuentes proteicas de AC. El análisis de variables se realizó con programa STATA versión 13. Se aplicó prueba de chicuadrado, ANOVA one way. Resultados: Se estudiaron 85 lactantes, 54\% sexo femenino, $68 \%$ tuvo LM exclusiva hasta los 6 meses, $84,7 \%$ inició AC a esa edad. 37,6\% cumplieron la incorporación de pescado, $49,4 \%$ con incorporación de legumbres y $45,8 \%$ cumplieron con huevo. Tuvieron mayor consumo de carne tipo vacuno, pollo o pavo en 52\% lactantes 10-12 meses, 83\% lactantes 13-24 meses, asociándose con ser el primer hijo y mayor edad, al igual que para el huevo en que mayores de 12 meses comían más de lo recomendado. Encontramos bajo cumplimiento para el pescado $78 \%$ lactantes 10-12 meses, de los cuales 35\% nunca habían comido, 83,2\% lactantes 13-24 meses los que
Palabras clave: Lactancia Materna; Alimentación Complementaria; Proteínas Dietarias; Recomendaciones Dietarias

Correspondencia:

Catalina Le Roy

catalinaleroy@yahoo.es 
2,7\% nunca había comido. De 45 lactantes alimentados sólo con FL más AC, 28 recibían $\geq 1$ g/k/día de proteínas por concepto de FL. Conclusión: La mayoría de los lactantes cumplieron con LM exclusiva hasta los 6 meses e inicio AC a esta edad. La mayoría incorporó tardíamente legumbres, huevo y pescado, existiendo excesivo consumo de carne de tipo vacuno, pollo o pavo y escaso de pescado, especialmente a mayor edad.

\begin{abstract}
Adequate nutrition includes breastfeeding, infant formula, and the incorporation of complementary feeding (CF). Objective: To describe compliance with the recommendations for protein intake from CF in healthy infants between 7 and 24 months old. Patients and Method: Healthy infants from an outpatient center were studied. To collect the variables under study, we designed and applied a questionnaire. We obtained demographic data, breastfeeding, use of formula, and incorporation and compliance with protein intake data. The analysis of variables was carried out with STATA software version 13, and the Chi-square test and one-way ANOVA were applied. Results: 85 infants were studied, $54 \%$ were female, $68 \%$ of infants were exclusively breastfed until 6 months old, and $84.7 \%$ started complementary feeding at that age. $37.6 \%$ complied with the incorporation of fish, $49.4 \%$ with legumes, and $45.8 \%$ with egg. $52 \%$ of infants aged between 10 and 12 months and $83 \%$ of infants between 13 and 24 months consumed more beef, chicken, or turkey. This data was associated with being the first child and older age, as well as egg consumption where infants older than 12 months ate more than recommended. We observed lower than recommended fish consumption in $78 \%$ of infants aged between 10 and 12 months and 83.2\% of infants between 13 and 24 months, of which $35 \%$ and $2.7 \%$ had never eaten fish, respectively. 45 infants were fed only with formula and complementary feeding, $28(62 \%)$ of them received $\geq 1 \mathrm{~g} / \mathrm{kg} /$ day of protein from infant formula. Conclusions: Most infants were exclusively breastfed until 6 months old and incorporated complementary feeding at that age. Most infants incorporated legumes, eggs, and fish later than recommended, and there was excessive consumption of beef, chicken, or turkey and little consumption of fish, especially at older ages.
\end{abstract}

Keywords:

Breastfeeding;

Complementary

Feedings;

Dietary Proteins;

Recommended Dietary

Allowances

\section{Introducción}

Durante los dos primeros años de vida se realiza una transición desde alimentación láctea exclusiva hacia alimentación mixta basada en lácteos y alimentos sólidos, incorporándose a la mesa familiar ${ }^{1}$. Las principales fuentes proteicas en este período son: lácteos (leche materna (LM) y/o fórmulas lácteas (FL)), carnes (vacuno, pollo, pavo, cerdo, pescado), leguminosas y huevo $^{1,2}$.

Las recomendaciones de ingesta son para no presentar déficit ni excesos de proteínas y otros nutrientes importantes en esta etapa de rápido crecimiento, contenidos naturalmente en esas fuentes proteicas o en lácteos; éstos son hierro, zinc, vitamina B12 y ácidos grasos esenciales ${ }^{3}$.

En el año 2010 se realizó una encuesta nacional de consumo alimentario (ENCA), que incluyó a población desde 2 años de edad. Nacionalmente destacó que sólo el 13,7\% cumplía las recomendaciones de consumo de las guías nacionales, siendo pescado, lácteos y leguminosas aquellos con menor cumplimiento. En el grupo entre 2 y 5 años destacó la baja ingesta de pescado $(21,5 \%)$ y leguminosas $(31 \%)^{4}$.

Es tema actual de estudio la asociación entre inges- ta proteica y exceso de peso, encontrándose que aquellos lactantes menores de un año alimentados con FL con mayor contenido proteico que LM poseen mayor riesgo de presentar obesidad en edad escolar, esto aún no está claro para el segundo año de vida ${ }^{5,6}$.

El objetivo principal de este estudio es describir el cumplimiento de las recomendaciones de la guía nacional de alimentación normal vigente ${ }^{1}$, en relación a la incorporación de las distintas fuentes proteicas y su frecuencia de consumo en lactantes sanos entre 7 y 24 meses.

\section{Pacientes y Método}

Estudio de tipo transversal con reclutamiento prospectivo. Se incluyeron lactantes sanos de 7 a 24 meses atendidos en el centro de atención ambulatorio San Joaquín de Red Salud UC Christhus, entre febrero de 2019 a marzo de 2020. Se excluyeron niños que presentaban alguna patología que afecte o restrinja su alimentación como alergia alimentaria, uso de sonda nasogástrica o gastrostomía, insuficiencia renal o hepática, enfermedades metabólicas, opciones vegetarianas, prematuros e hijos de inmigrantes que pueden 
mantener esquemas de alimentación de su nacionalidad.

Se confeccionaron cuestionarios para obtener la siguiente información: fecha de nacimiento, sexo, semanas de gestación y peso al nacer, asistencia a sala cuna, orden de hijo, responsable de la alimentación, familia extendida viviendo en el mismo hogar (familiares diferentes a padres y hermanos), edad y escolaridad materna. Se obtuvo de la ficha electrónica el peso y talla más reciente realizándose el diagnóstico nutricional según los estándares de crecimiento OMS y definiciones ministeriales ${ }^{7}$.

Se consultó sobre duración de LM exclusiva (LME), duración total de LM, edad de incorporación de FL, FL utilizada, cantidad de fórmula consumida por día y su preparación. La fórmula utilizada se clasificó en fórmula de inicio, de continuación, del programa nacional de alimentación complementaria (PNAC) para menor de 18 meses (Purita Fortificada), fórmula comercial para mayor de 1 año, fórmula del PNAC para mayores de 18 meses (Purita Cereal) y en otras aquellas fórmulas que contenían algún cambio específico de sus componentes. En el caso de los niños que utilizaban sólo FL al momento del estudio, se calculó los g/kg/día de proteínas consumido por ésta.

La tabla 1 resume las recomendaciones de alimentación complementaria (AC) según la guía nacional de alimentación de 2016, a partir de ellas se definieron 3 grupos: grupo I (GI) entre 7-9 meses, grupo II (GII) 10-12 y grupo III (GIII) 13-24 meses, de acuerdo a la edad recomendada para iniciar cada fuente proteica: carne de vacuno o pollo o pavo o cerdo, pescado, legumbres, huevo y la frecuencia a consumir cada uno de ellos en la semana ${ }^{1}$. De esta forma se determinó si los alimentos se incorporaron adecuados, precoz o tardíamente y si cumple con las recomendaciones de frecuencia semanal de consumo de cada uno de ellos.

Si bien este estudio se puede considerar exploratorio, realizamos un cálculo de tamaño muestral a partir del número de niños incluidos en la ENCA, en la cual, la muestra de niños encuestados de 2 a 5 años fue de 257, que subdivididos en 3 grupos según edad resulta un total de 85 sujetos/año, por lo cual al estudiar aproximadamente solo un tramo etario calculamos un total de 85 sujetos, los cuales se fueron reclutando en forma prospectiva, completando número similar entre los tres grupos definidos.

Se analizaron los datos por el programa STATA versión 13, las variables categóricas se describen como frecuencia relativa y absoluta. Se realizó prueba de chi-cuadrado en asociaciones de categorización de inicio y cumplimiento de recomendaciones de alimentación con edad materna mayor o menor a 30 años, ingesta proteica por FL mayor o menor 1 $\mathrm{g} / \mathrm{kg} / \mathrm{d}$ áa, peso de nacimiento para edad gestacional, escolaridad materna, orden del número de hijo, asistencia a sala cuna, responsable de la preparación de alimentos. Se realizó ANOVA one way para comparar los g/kg/día de proteínas desde FL entre los 3 grupos de edades. Se consideró una significancia estadística con $\mathrm{p}<0,05$.

Esta investigación tiene aprobación del comité de ética de la Facultad de Medicina de la Pontificia Universidad Católica de Chile, número 180322008. Se obtuvo el consentimiento informado por escrito de los padres antes del ingreso al estudio.

\section{Resultados}

Se estudiaron 85 sujetos, la tabla 2 muestra sus datos y variables demográficas. Destaca que $54 \%$ eran de sexo femenino, ninguno presentaba talla baja, 54\% eran el primer hijo y en $70 \%$ la responsable de la alimentación era la madre. Recibieron LME hasta los 6 meses 68\%. De los mayores de 12 meses seguían LM un $54,9 \%$. Iniciaron $\mathrm{FL} \leq 6$ meses $44,6 \%$ y $21 \%$ en mayor de un año. De los lactantes alimentados sólo con FL más AC, $62 \%$ recibían $\geq 1 \mathrm{~g} / \mathrm{kg} /$ día de proteínas por FL. En la tabla 3 se muestra la ingesta láctea según los grupos de estudio, sin diferencia significativa en la ingesta promedio de gramos de proteínas $/ \mathrm{kg} /$ día como FL $(\mathrm{p}=0,2)$.

Iniciaron $\mathrm{AC}$ a los 6 meses $84,7 \%$, carne de vacuno, pollo o pavo o cerdo $\leq 7$ meses el $90,5 \%$, pescado $\leq 8$ meses $37,6 \%$, legumbres $\leq 9$ meses $49,4 \%$ y huevo $\leq 11$ meses $45,8 \%$. La figura 1 muestra la incorporación precoz, tardía, adecuada o sin incorporación de cada fuente proteica; se representa como "no aplica" aquellos que aún no tenían la edad recomendada para incorporarlos.

Cumplieron recomendación de inicio carne de vacuno, pollo o pavo o cerdo a los 6 meses 22/26 del GI, 18/23 del GII y 21/36 del GIII. Respecto al pescado,

Tabla 1. Recomendaciones de incorporación y consumo semanal de fuentes proteicas en alimentación complementaria en lactantes ${ }^{1}$

\begin{tabular}{|c|c|c|c|c|}
\hline \multirow[t]{2}{*}{ Alimento } & \multirow{2}{*}{$\begin{array}{l}\text { Inicio } \\
\text { (mes) }\end{array}$} & \multicolumn{3}{|c|}{ Recomendación (veces/semana) } \\
\hline & & $\begin{array}{c}\mathrm{Gl} \\
\text { (7-9 meses) }\end{array}$ & $\begin{array}{c}\text { GII } \\
\text { (10-12 meses) }\end{array}$ & $\begin{array}{c}\text { GIII } \\
\text { (13-24 meses) }\end{array}$ \\
\hline Carne* & 6 & $3-7$ & 3 & 2 \\
\hline Pescado & $6-7$ & $0-2$ & 2 & 2 \\
\hline Legumbre & $7-8$ & $0-2$ & 2 & $1-2$ \\
\hline Huevo & 9-10 & $0-2$ & $0-2$ & $1-2$ \\
\hline
\end{tabular}

*Carne de vacuno o pollo o pavo o cerdo. 
Tabla 2. Variables y datos demográficos de lactantes estudiados

\begin{tabular}{|c|c|}
\hline Variable & Frecuencia n (\%) \\
\hline $\begin{array}{l}\text { Edad } \\
\text { Grupo I } \\
\text { Grupo II } \\
\text { Grupo III }\end{array}$ & $\begin{array}{l}26(31) \\
23(27) \\
36(42)\end{array}$ \\
\hline $\begin{array}{l}\text { Sexo } \\
\text { Femenino } \\
\text { Masculino }\end{array}$ & $\begin{array}{l}46(54) \\
39(46)\end{array}$ \\
\hline $\begin{array}{l}\text { Adecuación PN } \\
\text { PEG } \\
\text { AEG } \\
\text { GEG }\end{array}$ & $\begin{array}{r}6(7) \\
68(80) \\
11(13)\end{array}$ \\
\hline $\begin{array}{l}\text { Diagnóstico nutricional } \\
\text { Riesgo de desnutrición } \\
\text { Eutrofia } \\
\text { Sobrepeso } \\
\text { Obesidad }\end{array}$ & $\begin{array}{r}2(3) \\
47(61) \\
17(22) \\
11(14)\end{array}$ \\
\hline $\begin{array}{l}\text { Edad materna } \\
\text { 20-30 años } \\
\text { 31-43 años }\end{array}$ & $\begin{array}{l}35(41) \\
50(59)\end{array}$ \\
\hline $\begin{array}{l}\text { Número de hijo } \\
\text { Primero } \\
\text { Segundo } \\
\text { Tercero }\end{array}$ & $\begin{array}{r}46(55) \\
31(36) \\
8 \quad(9)\end{array}$ \\
\hline $\begin{array}{l}\text { Asistencia a sala cuna } \\
\text { No } \\
\text { Sí }\end{array}$ & $\begin{array}{l}66(78) \\
19(22)\end{array}$ \\
\hline $\begin{array}{l}\text { Familia extendida } \\
\text { No } \\
\text { Sí }\end{array}$ & $\begin{array}{l}62(73) \\
23(27)\end{array}$ \\
\hline $\begin{array}{l}\text { Responsable de alimentac } \\
\text { Madre } \\
\text { Abuelos } \\
\text { Asesora del hogar } \\
\text { Otro familiar cercano } \\
\text { Sala cuna }\end{array}$ & $\begin{array}{r}60(71) \\
11(13) \\
1 \quad(1) \\
1 \quad(1) \\
12(14)\end{array}$ \\
\hline $\begin{array}{l}\text { Escolaridad materna } \\
\text { Media completa } \\
\text { Superior completa }\end{array}$ & $\begin{array}{l}18(21) \\
67(78)\end{array}$ \\
\hline
\end{tabular}

PN: peso de nacimiento; EG: edad gestacional; PEG: pequeño para la edad gestacional; AEG: adecuado para la edad gestacional; GEG: grande para la edad gestacional. Grupo I: 7-9 meses; Grupo II: 10-12 meses; Grupo III: 13-24 meses. cumplieron recomendación de inicio 4/26 GI, 3/23 del GII, 6/36 del GIII. Destaca que nunca habían comido pescado 21/26 de GI y 8/23 del GII.

Por otra parte, cumplieron incorporación de legumbre: 6/26 del GI, 10/23 del GII, 13/36 del GIII. Cabe destacar que del GI 5/26 lactantes no cumplían edad para incorporación de legumbres por lo que no se les consideró en este ítem de cumplimiento, mientras que 15/21 tenía edad para incorporación y aún no lo hacía. Incorporaron precozmente las legumbres 7/36 del GIII y 16/36 de este grupo lo hace tardíamente.

Cumplieron inicio de huevo: 9/23 del GII y 13/36 del GIII. Incorporaron precozmente 6/23 del GII y 9/36 del GIII, por el contrario, tardíamente 3/26 del GII y 16/36 del GIII. Nunca habían comido huevo 5/23 del GII. La figura 2 muestra el cumplimiento de frecuencia de consumo de cada alimento para los grupos II y III.

Se buscó asociación entre variables de adecuación del peso para edad gestacional, edad materna, escolaridad materna, asistencia a sala cuna, diagnóstico nutricional del niño, grupo etario, orden de hijo, duración de LME, LM total, inicio de FL con el inicio de papillas, inicio y cumplimento de las recomendaciones para cada uno de las fuentes proteicas estudiadas (carne tipo vacuno o pollo o pavo o cerdo, legumbres, pescado y huevo) encontrándose que quienes eran el primer hijo consumían más carne que lo recomendado $(\mathrm{p}=0,01)$, lo que también se encontró en los grupos de mayor edad ( 10 a 24 meses) ( $p<0,001)$, para huevo los mayores de 12 meses comían más de lo recomendado ( $\mathrm{p}<0,001$ ), el GII tuvo una menos ingesta de legumbres menor de lo recomendado $(p<0,001)$ y para todos los grupos era menor la ingesta de pescado $(\mathrm{p}<0,001)$. No se encontró asociación entre diagnóstico nutricional con la fuente láctea utilizada o los $\mathrm{g} / \mathrm{kg} /$ día de proteínas de FL, en aquellos en que correspondía hacer el cálculo.

Se encontró que aquellos que no asisten a sala cuna cumplían con más frecuencia la recomendación de ingesta de legumbres $(\mathrm{p}=0,006)$.

Tabla 3. Frecuencia y porcentaje de alimentación láctea según grupo de estudio

\begin{tabular}{|c|c|c|c|c|}
\hline \multicolumn{2}{|c|}{ Alimentación láctea } & Gl $n=26$ & GII $n=23$ & GIII $n=36$ \\
\hline \multicolumn{2}{|c|}{ Leche materna $(\%)$} & $13(50)$ & $7(30,4)$ & $5(13,8)$ \\
\hline \multicolumn{2}{|c|}{ Mixto (LM + LA), n (\%) } & $2(7,6)$ & $2(8,6)$ & $11(30,5)$ \\
\hline Uso fórmula & $\begin{array}{l}\text { Fórmula de inicio } \mathrm{n}(\%) \\
\text { Fórmula de continuación } \mathrm{n}(\%) \\
\text { Fórmula del PNAC } \mathrm{n}(\%) \\
\text { Fórmula comercial para > } 1 \text { años, n (\%) } \\
\text { Otras }\end{array}$ & $\begin{array}{l}2(7,6) \\
3(11,5) \\
2(7,6) \\
1 \quad(3,8) \\
3(11,5)\end{array}$ & $\begin{array}{l}1(4,34) \\
3(13,0) \\
4(17,3) \\
4(17,3) \\
2(8,6)\end{array}$ & $\begin{array}{c}1(2,7) \\
7(19,4) \\
12(33,3)\end{array}$ \\
\hline \multicolumn{2}{|c|}{ g proteína/k/día* (promedio, DS) } & $1,3 \pm 0,8$ & $1,15 \pm 0,6$ & $0,9 \pm 0,4$ \\
\hline
\end{tabular}

*Calculado sólo para aquellos lactantes con fórmula láctea y sin leche materna. LM: leche materna; LA: lactancia artificial; PNAC: programa nacional de alimentación complementaria. Grupo I (GI): 7-9 meses; Grupo II (GII): 10-12 meses; Grupo III (GIII): $13-24$ meses. 

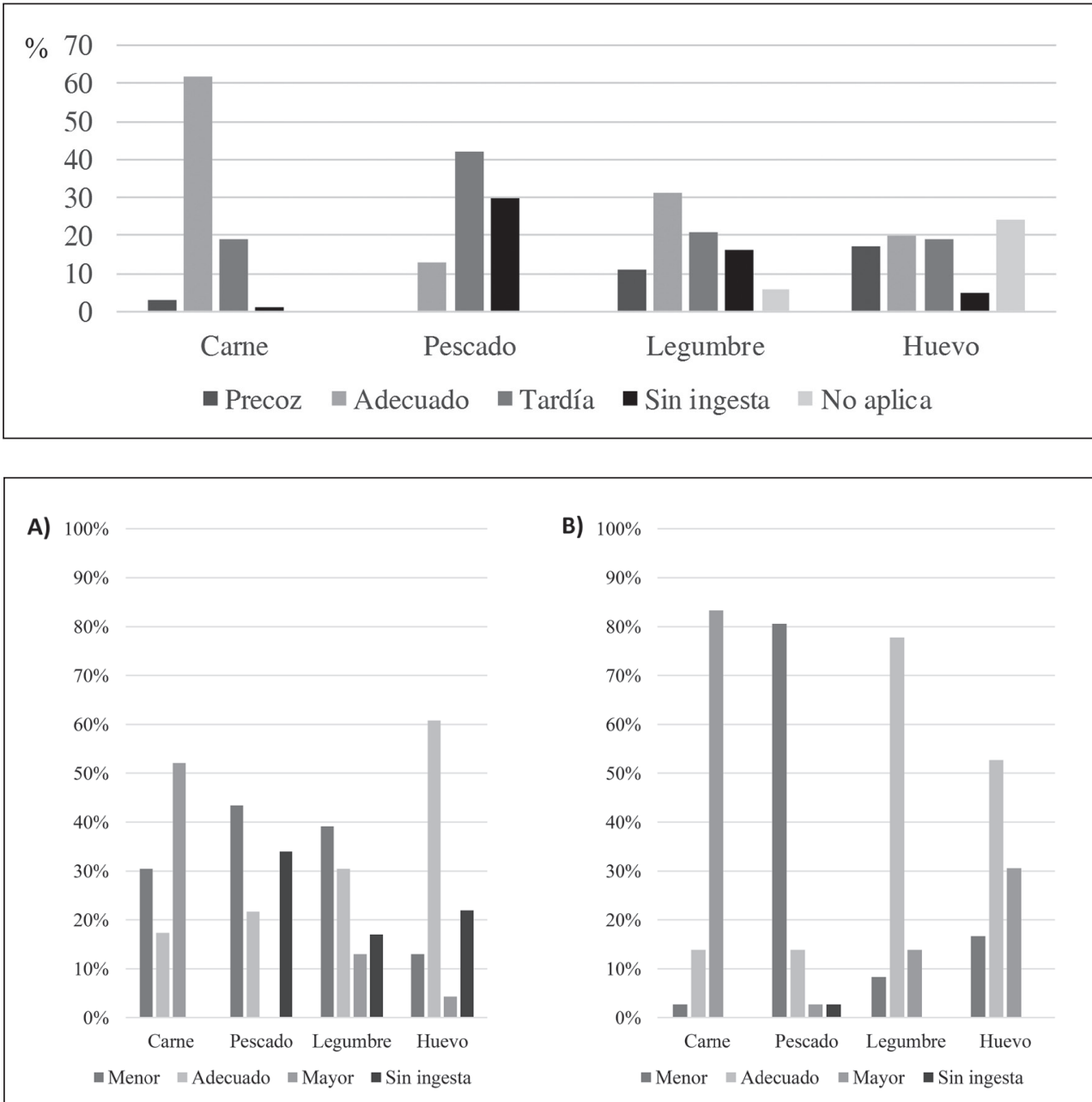

\section{Discusión}

La alimentación en los primeros 1.000 días de vida constituye uno de los principales determinantes del crecimiento y desarrollo en la infancia ${ }^{8}$. En este periodo de tiempo, los lactantes reciben proteínas de distintas fuentes proteicas contenidas en la LM, FL y $\mathrm{AC}^{6}$.

Se recomienda LM hasta los 6 meses por sus beneficios tanto en el niño como la madre 9 . En Chile, la prevalencia de LME al sexto mes entre el año 2005 a 2014 fue de $51 \%{ }^{10}$. En nuestro estudio un gran porcentaje tuvo LME hasta los 6 meses, sin encontrar asociaciones significativas entre escolaridad materna y cumplimiento de esta recomendación, a diferencia un estudio realizado en 2012 en nuestro país, donde se encontró que, entre otros factores, la menor escolaridad materna se relacionaba más con LME hasta los 6 meses $^{11}$.

Dado que la LM aporta energía y factores inmunológicos los primeros dos años de edad, se recomienda extenderla hasta los dos años de vida o más, mientras se cumpla con una adecuada AC y buen estado nutri- cional $^{2}$. De los lactantes estudiados mayores de un año, más de la mitad recibía LM.

Se sabe que los nutrientes aportados por LM difieren de los proporcionados por FL, especialmente en la cantidad de proteínas ${ }^{3}$. Se ha encontrado asociación entre exceso de peso en etapa escolar y alimentación con FL con mayor contenido proteico en el primer año de vida ${ }^{5,12}$, a su vez, una revisión sistemática, basada en 4 estudios controlados aleatorios, concluye que el uso de FL con baja concentración de proteínas (similar a LM) lleva a un adecuado incremento de peso y talla ${ }^{13}$. Si bien en nuestro estudio hubo alto porcentaje de lactantes alimentados con LM, más de la mitad usaban exclusivamente fórmula de inicio o continuación para su alimentación láctea, sin encontrarse asociación con exceso de peso. Al cuantificar la ingesta proteica por FL encontramos que un gran porcentaje recibía $\geq 1 \mathrm{~g} / \mathrm{kg} /$ día de proteínas aportados sólo por ésta, excediendo lo recomendado por la OMS, que establece como nivel seguro de ingesta proteica $1 \mathrm{~g} / \mathrm{kg} / \mathrm{día}$, los que deberían ser aportados por distintas fuentes proteicas. No encontramos asociaciones significativas entre esta ingesta 
proteica y diagnóstico nutricional, para lo cual sería recomendable un estudio con mayor seguimiento de los sujetos; sin embargo en base a la evidencia, actualmente se están realizando estudios para evaluar si se puede reducir de forma segura la cantidad de proteínas en la fórmula láctea ${ }^{3}$. A pesar de que varios estudios asocian positivamente la cantidad de proteínas de FL con mayor ganancia de peso y mayor IMC a largo plazo, el efecto de mayor consumo de proteínas proveniente de diversas fuentes (lácteas más AC) no está claro, encontrándose resultados mixtos ${ }^{6,14}$.

Desde los 6 meses de edad se debe iniciar la AC porque la LM no cubre todas las necesidades del niño principalmente con la ingesta de micronutrientes como hierro, zinc, ácidos grasos esenciales ${ }^{3}$. Un estudio mexicano encontró que la edad de inicio de AC fue en promedio a los 6,1 meses $^{15}$, lo que es concordante con nuestros resultados y se encontró asociación estadísticamente significativa con diagnóstico nutricional eutrofia, mientras que, un estudio realizado en 8 países europeos, muestra que lactantes que iniciaron $\mathrm{AC} \geq 7$ meses, tuvieron mayor prevalencia de exceso de peso en la infancia ${ }^{16}$.

Durante los últimos años, ha existido un gran cambio en la incorporación de los alimentos aquí estudiados ya que, a pesar de introducir tardíamente alimentos potencialmente alergénicos, la prevalencia de alergias alimentarias sigue aumentando ${ }^{17}$. Estudios mostraron que retrasar la introducción de éstos podría aumentar el riesgo de sensibilización a $\operatorname{IgE}$ y alergia alimentaria ${ }^{18}$. Actualmente, la evidencia sugiere que la introducción temprana de estos alimentos es una estrategia preventiva efectiva ${ }^{19}$. Un metaanálisis de 2016 que incluye 6 estudios, concluye que la introducción temprana del huevo ( 6 meses) está asociada con menor riesgo de alergia a éste ${ }^{20}$.

Llevará algún tiempo para lograr por parte de la población la incorporación de este tipo de alimentos desde los 7 meses, es más, encontramos que más de la mitad incorporó tardíamente el huevo, pescado y legumbres. No encontramos asociación entre incorporación tardía de estos alimentos con edad materna ni con la persona responsable de la alimentación del lactante.

Tanto en el GII como en GIII existe bajo cumplimiento de frecuencia de consumo de pescado, con mayor ingesta de carne, sobre todo para el grupo GIII, en que los resultados se asemejan a lo encontrado en grupo de 2 a 5 años y en la población general chilena de la ENCA, probablemente por la incorporación del niño a la mesa familiar ${ }^{4}$.

En cuanto a variabilidad dietaria, estudios del sur de Asia, identificaron como determinantes de prácticas inadecuadas de AC a la falta de educación materna y menor estrato socioeconómico ${ }^{21}$, esto es concordante con los hallazgos de la ENCA en nuestro país, donde se constata que el estrato socioeconómico alto tiene dieta más variada con mayor consumo de pescado y carne, mientras que, en los estratos socioeconómico bajos, mayor consumo de legumbres y bajo consumo de pescado. Si bien nosotros no estudiamos nivel socio- económico, destaca el bajo cumplimiento de recomendación de pescado, tanto en la introducción a los 8 meses como en la frecuencia de consumo semanal.

Dentro de las limitaciones de nuestro estudio consideramos que estos resultados no se pueden generalizar para la población total chilena, sin embargo, constituyen una primera aproximación a este grupo etario. Otra limitación es que, al ser un estudio de tendencia de consumo, no se puede hacer una medición de la ingesta proteica total del niño.

Nuestros resultados son un primer aporte para describir la ingesta alimentaria para los lactantes, lo que permitiría identificar en qué puntos hacer énfasis en las recomendaciones de alimentación en la práctica diaria de nuestros controles sano.

\section{Conclusiones}

En la población estudiada la gran mayoría cumple la recomendación de LME a los 6 meses y el inicio de AC. La incorporación de los alimentos en general es tardía para legumbres, huevo y sobre todo pescado, así como el adecuado cumplimiento de ellos dentro de su alimentación habitual, siendo para estos alimentos enumerados menos de la recomendación y el consumo de carnes de vacuno o pollo o pavo o cerdo mayor que la recomendación, lo cual se va asemejando a la población general chilena como al grupo de 2 a 5 años que sí ha sido estudiado en Chile. Hubo algunas asociaciones entre las variables estudiadas y el inicio y cumplimiento de las recomendaciones de ingesta siendo importante seguir estudiándolas con grupos más representativos de nuestro país y relacionándolas con algunos efectos a largo plazo como son las enfermedades alérgicas y exceso de peso.

\section{Responsabilidades Éticas}

Protección de personas y animales: Los autores declaran que los procedimientos seguidos se conformaron a las normas éticas del comité de experimentación humana responsable y de acuerdo con la Asociación Médica Mundial y la Declaración de Helsinki.

Confidencialidad de los datos: Los autores declaran que han seguido los protocolos de su centro de trabajo sobre la publicación de datos de pacientes.

Derecho a la privacidad y consentimiento informado: Los autores han obtenido el consentimiento in- 
formado de los pacientes y/o sujetos referidos en el artículo. Este documento obra en poder del autor de correspondencia.

\section{Conflicto de intereses}

Los autores declaran no tener conflicto de intereses.

\section{Referencias}

1. Departamento de Nutrición y Alimentos, División de Políticas Públicas Saludables y Promoción, Subsecretaría de Salud Pública del Ministerio de Salud. Guía de alimentación del niño(a) menor de 2 años, guía de alimentación hasta la adolescencia, $4^{\circ}$ edición, 2015. http:// www.crececontigo.gob.cl/wp-content/ uploads/2016/01/Guia-alimentacionmenor-de-2.pdf. Enero de 2019.

2. Organización panamericana de la salud, organización mundial de la salud. Unidad de nutrición, salud de la familia y comunidad. Principios de orientación para la alimentación complementaria del niño amamantado entre 6-24 meses de edad. 2002 https:// www.who.int/maternal_child_adolescent/ documents/9241593431/es/. Última visita: enero de 2019.

3. Fewtrell M, Bronsky J, Campoy C, et al. Complementary feeding: A position paper by the European Society for paediatric, Gastroenterology, Hepatology, and Nutrition (ESPGHAN) Committee on Nutrition. J Pediatr Gastroenterol Nutr 2017;64:119-32.

4. Departamento de Nutrición, Escuela de Nutrición, Escuela de Salud Pública, Facultad de Medicina, Centro de Microdatos, Facultad de Economía y Negocios. Universidad de Chile. Encuesta nacional de consumo alimentario. Informe final. 2010. https://www.minsal. cl/sites/default/files/ENCA-INFORME_ FINAL.pdf. Última visita: enero de 2019.

5. Koletzko B, Von Kries R, Closa R, et al. Lower protein in infant formula is associated with lower weight up to age 2 $y:$ A randomized clinical trial. Am J Clin Nutr 2009;89:1836-45.

6. Tang M. Protein intake during first two years of life and its association with growth and risk of overweight. Int J Environ Res Public Health 2018;15:1-8.
7. Subsecretaría de Salud Pública. División Políticas Públicas Saludables y Promoción. Departamento de Nutrición y Alimentos. Ministerio de Salud. Patrones de crecimiento para la evaluación nutricional de niños, niñas y adolescentes desde el nacimiento hasta los 19 años de edad.2018. http://www. bibliotecaminsal.cl/wp/wp-content/ uploads/2018/03/2018.03.16-Patrones-decrecimiento-para-la-evaluaci\%C3\%B3nnutricional-de-ni\%C3\%Blosni\%C3\%B1as-y-adolescentes-2018.pdf. Última visita: enero de 2019.

8. Department of Nutrition for Health and Development-World Health Organization. Essential nutrition actions: Improving maternal, newborn, infant and young child health and nutrition. 2013. http://apps.who.int/iris/ bitstream/10665/84409/1/978924150555_ eng.pdf Última visita: enero de 2019.

9. Shamir R, Koletzco B, Agostoni C, et al, ESPGHAN`s 2008 recommendation for early introduction of complementary foods: how good is the evidence? Matern Child Nutr 2012;15:139-40.

10. Departamento de Nutrición y Alimentos, División de Políticas Públicas Saludables y Promoción, Subsecretaría de Salud Pública del Ministerio de Salud. Vigilancia del estado nutricional de la población bajo control y de la lactancia materna en el sistema público de salud de Chile. 2014. https://www.minsal.cl/sites/default/ files/files/Informe $\% 20$ Vigilancia $\% 20$ Nutricional\%20y\%20Lactancia\%20 Materna\%20Diciembre\%202014.docx. pdf. Última visita enero de 2019.

11. Nino R, Silva G, Atalah E. Factores asociados a lactancia materna exclusiva. Rev Chil Pediatr 2012;83:161-9.

12. Heinig M, Nommsen L, Peerson J, et al. Energy and protein intakes of breast-fed and formula-fed infants during the first year of life and their association with growth velocity: The darling study. Am J Clin Nutr 1993;58:152-61.
13. Patro- Golab B, Zalewski B, Kowenhoven $\mathrm{S}$, et al. Protein concentration milk formula growth, and later risk of obesity: A systematic Review. J Nutr 2016;146:55164.

14. Tang M, Krebs NF. High protein intake from meat as complementary food increases growth but not adiposity in breastfed infants: a randomized trial. Am J Clin Nutr 2014;100:1322-8.

15. Ortega C, Vidaña $D$, Basto A, et al. Complementary feeding practices in Mexican healthy infants: How close are they to current guidelines? Bol Med Hosp Infant Mex 2019;76:265-72.

16. Papoutsou S, Savva S, Hunsberguer M, et al. Timing of solid food introduction and association with later childhood overweight and obesity: The IDEFICS study. Matern Child Nutr 2018;14:12471. https://doi.org/10.1111/mcn.12471.

17. Prescott S, Pawankar R, Allen K, et al. A global survey of changing patterns of food allergy burden in children. World Allergy Organ J 2013;6:1-12.

18. Isse I, Tromp M, Kiefete J, The introduction of allergenic foods and development of reported wheezing and eczema in childhood. The generation R study. Arch Pediatr Adolesc Med 2011;165:933-8.

19. Perkin M, Logan K, Marrs T, et al. Enquiring early about tolerance (EAT) study: feasibility of an early allergenic food introduction regimen. J Allergy Clin Immunol 2016;137:1477-86

20. Despolerodiakonou M, García V, Logan A, et al. Timing of allergenic food introduction to the infant diet and risk of allergic or autoimmune disease. A Systematic review and Meta-analysis. JAMA 2016;316:1181-92.

21. Semarath U, Kinsgley E, Dur-e- Samin A, et al. Comparisons of complementary feeding indicators and associated factors in children aged 6-23 months across five South Asian countries. Matern Child Nutr 2011;8:89-106 